\title{
Laponita: una nanotecnología que retarda la licuefacción
}

\author{
Laponite: a nanotechnology that retards liquefaction
}

Fecha de entrega: 7 de diciembre 2016 Fecha de aceptación: 9 de mayo 2017

\section{Felipe Ochoa-Cornejo y Sergio Muñoz}

Departamento de Ingeniería Civil, Universidad de Chile, Av. Blanco Encalada 2002, Santiago, Chile, fochoa@ing.uchile.cl, smunoz@ing.uchile.cl

La licuefacción de depósitos de arena suelta ha causado daños significativos en eventos sísmicos de gran magnitud, tanto en Chile (Valdivia 1960, Valparaíso 1985, Maule 2010), como en otros países (Nueva Zelanda 2010, Japón 2011). Es por esto que se requiere el desarrollo de métodos de mejora del suelo para mitigar la licuefacción, en particular en terrenos cercanos a estructuras existentes, donde no pueden aplicarse mejoramientos tradicionales de densificación. En este contexto, este trabajo presenta un estudio experimental que explora el uso de Laponita, una nano-arcilla sintética de diámetro diez veces más pequeño que la Bentonita, para el tratamiento de suelos licuables. Se presentan resultados de ensayos triaxiales cíclicos para estudiar el efecto de 1\% de Laponita (por peso seco de arena) sobre la resistencia a la licuefacción de arena limpia con densidad relativa entre 15 y 25\%. Se observa que la presencia de 1\% de Laponita mejora la resistencia con respecto a arenas limpias, aumentando el número de ciclos para la licuefacción hasta dos órdenes de magnitud, bajo la misma razón de tensión cíclica. Este efecto es comparable al inducido por Bentonita, pero con menos material. Además, se abordan los mecanismos detrás de la mejora gracias al fluido tixotrópico formado entre los poros de arenas.

Palabras clave: Laponita, Bentonita, nanomateriales, arena, licuefacción
Earthquake induced liquefaction of loose sand deposits has caused significant damage in severe seismic events in Chile (Valdivia 1960, Valparaíso 1985, Maule 2010) and in other countries (New Zealand 2010, Japón 2011). This requires the development of soil improvement methods to increase the liquefaction resistance of these deposits, particularly in proximity to existing structures, where traditional approaches relying on densification may not work. This paper presents an experimental study that explores the use of Laponite, a synthetic nano-clay with particle diameter ten times smaller than Bentonite, for treating liquefiable soils. The effect of the presence of a small amount of laponite $(1 \%$ by dry mass of the sand) on the liquefaction resistance of clean sand specimens with relative density in the 15-25\% range is studied through laboratory triaxial cyclic tests. The addition of $1 \%$ Laponite leads to a significant increase in liquefaction resistance, with respect to the clean sand, with the number of cycles to liquefaction increasing by approximately two orders of magnitude under the same cyclic stress ratio. This is comparable to the effect recently reported for Bentonite, but with much smaller addition of the nano-clay. The study addresses the mechanisms that explain the improvement based on the thixotropic fluid formed in the sand pore space.

Keywords: Laponite, Bentonite, nanomaterials, sand, liquefaction

\section{Introducción}

La licuefacción es un fenómeno catastrófico, de importancia primaria para la ingeniería chilena, el cual ocurre en depósitos granulares sueltos, y saturados, durante terremotos de gran magnitud. Durante el movimiento sísmico, el exceso de presión de poros disminuye la tensión efectiva del suelo. Cuando el exceso de presión de poros aumenta hasta igualar el confinamiento efectivo inicial, comienza la licuefacción, causando grandes deformaciones en el suelo. El terremoto del Maule 2010 evidenció los grandes daños que puede causar la licuefacción; se produce asentamiento y colapso de estructuras construidas sobre depósitos saturados de arenas sueltas, principalmente en sectores costeros. Debido a los severos daños observados en este, y otros terremotos, los esfuerzos se han concentrado en desarrollar métodos eficaces para aumentar 
la resistencia del suelo a la licuefacción. Esto se ha intentado, en particular, en terrenos cercanos a estructuras civiles (Gallagher y Mitchell, 2002), donde las soluciones de mejoramiento de suelos más tradicionales, basadas en la densificación, no son viables. En este contexto, el reciente método basado en la modificación del fluido intersticial de la arena, reemplazando el agua en los poros del material granular por una suspensión concentrada de Bentonita, ha demostrado promisorios resultados (El Mohtar et al., 2013, 2014).

La idea de usar Bentonita para mejorar un suelo, y mitigar la licuefacción, está respaldado por el positivo efecto de los finos plásticos en la resistencia a la licuefacción de arenas. Observaciones en laboratorio y terreno han demostrado que la presencia de finos plásticos en la matriz de arenas previene la licuefacción, aumentando el número de ciclos para gatillarla, gracias a la plasticidad de los finos (Troncoso y Verdugo, 1985; Ishihara, 1993; Polito y Martin, 2001; Ishihara y Koseki, 1989; Koester, 1994; Youd et al., 2001). Este efecto se observa indirectamente en el gráfico de resistencia a la licuefacción, a partir del número normalizado $\left(N_{1}\right)_{60}$ del ensayo de penetración estándar SPT, mostrado en la Figura 1. Este gráfico muestra que la resistencia de licuefacción CRR de arenas aumenta con el contenido de finos.

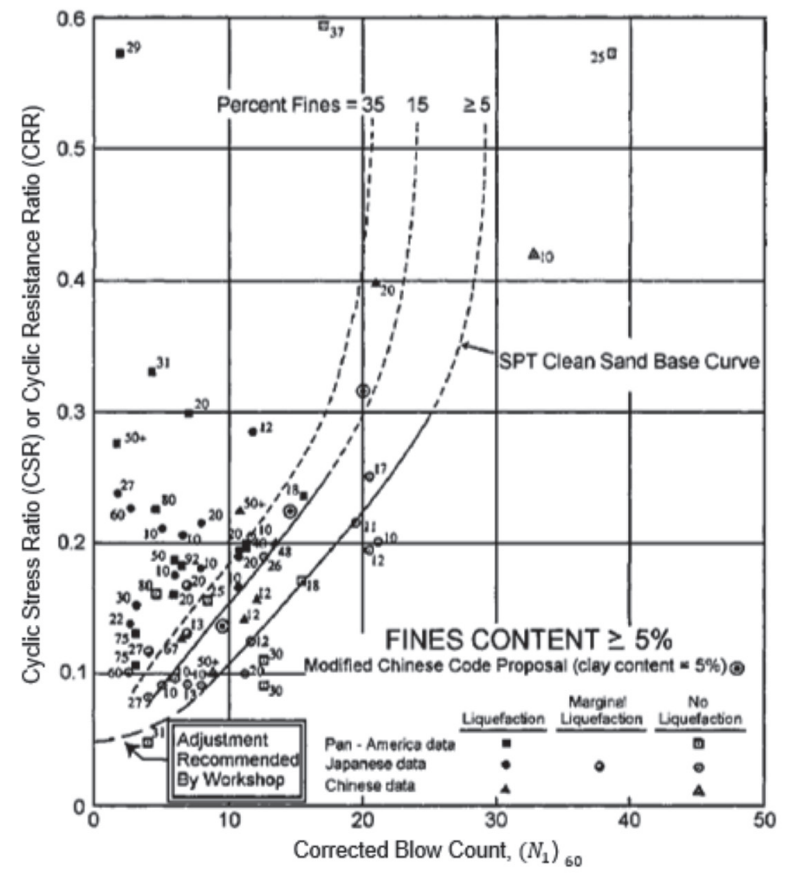

Figura 1: Influencia del contenido de finos en la licuefacción de arenas (Youd et al., 2001)
El positivo efecto que los finos de alta plasticidad, particularmente la Bentonita, tienen en la resistencia a la licuefacción de arenas ha sido documentado por El Mohtar et al. $(2013,2014)$, con ensayos triaxiales cíclicos, demostrando que $3 \%$ de Bentonita, por peso seco de arena, aumenta el número de ciclos para llegar a la licuefacción. Este aumento es, al menos, un orden de magnitud, en relación a muestras de arena seca, a una misma densidad relativa.

La Bentonita es un producto natural, y seguro, pero su aplicación es desafiante. La infiltración de una suspensión de Bentonita en el espacio intersticial de la arena requiere tratamiento químico con pyro-fosfato de sodio para modificar su reología de corto plazo (Clarke, 2008). Además, la infiltración de la Bentonita es difícil en depósitos de arena más finas. Esto, sumado a las impurezas de la Bentonita, las cuales pueden crear dificultades para alcanzar resultados consistentes en laboratorio.

Estas dificultades se pueden superar usando Laponita, una nano-partícula sintética con un diámetro diez veces más pequeño que la Bentonita y con un proceso de gelificación retardada natural (Mongondry et al., 2004). Estas características sugieren que la Laponita podría ser utilizada para tratar depósitos de arena fina, sin necesidad de modificación química. Además, la Laponita tiene un índice de plasticidad de 1200, casi el doble que la Bentonita, lo que requeriría menores porcentajes en relación a la Bentonita, para lograr la misma mejora en la resistencia de licuefacción.

Este estudio evalúa como la adición de 1\% de Laponita (por peso seco de arena) aumenta la respuesta cíclica de la arena de Ottawa. Las muestras se hacen mezclando Laponita con arena, en condición seca. Los resultados se comparan con datos publicados usando Bentonita.

\section{Programa experimental Materiales}

Los materiales utilizados para preparar las muestras de este estudio son arena limpia de Ottawa (C778) y Laponita RD. Respecto de la arena de Ottawa, sus partículas tienen un color gris claro con una forma redondeada a subredondeada. El coeficiente de uniformidad $C_{\mathrm{u}}$ es 1.48; el diámetro medio $D_{50}$ es de $0.4 \mathrm{~mm}$, con índice de vacío máximo y mínimo $e_{\max }=0.48 \mathrm{y} e_{\min }=0.78$ respectivamente. 
Su gravedad específica $G_{\mathrm{s}}$ es 2.65. En cuanto a la Laponita $\mathrm{RD}$, esta es una arcilla de esmectita sintética, con $1 \mathrm{~nm}$ de espesor y $25 \mathrm{~nm}$ de diámetro aproximadamente, un orden de magnitud menos que la Bentonita (Ruzicka y Zaccarelli, 2011). La estructura 2:1 de la Laponita consiste en una lámina dioctaédrica intercalada entre dos capas tetraédricas de silicio. El reemplazo parcial de iones de magnesio por iones de litio en la capa octaédrica resulta en una partícula con caras que presentan carga negativa, y un borde con cargas positivas. La Figura 2 muestra la estructura y composición química de la Bentonita y Laponita, comparando su tamaño de partícula. Para ponerlas en contexto, el peso específico de la Laponita es 2.57, y el de la Bentonita 2.65. La superficie específica de Laponita es de $470 \mathrm{~m}^{2} / \mathrm{g}$ (El Howayek, 2011), determinada con ensayos de absorción de vapor de agua usando el modelo de BET. Este valor es comparable a los $440 \mathrm{~m}^{2} / \mathrm{g}$ de la Bentonita usando la misma metodología (Money et al., 1952).

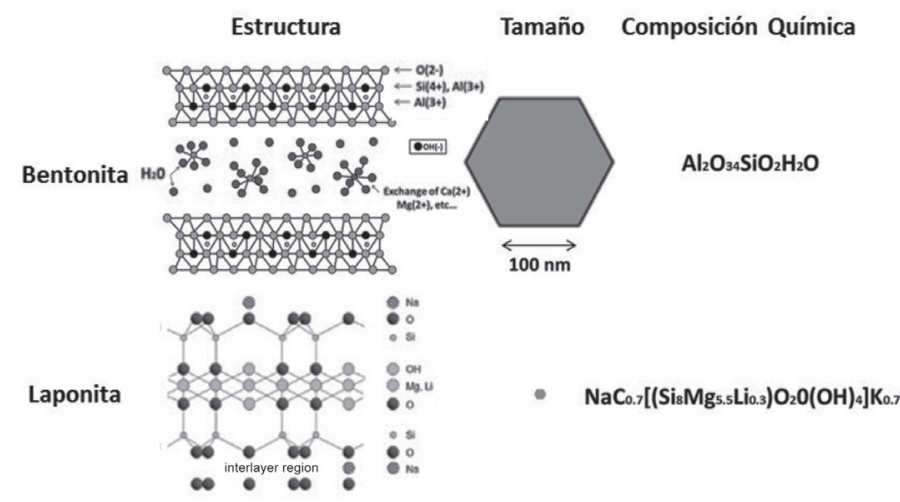

Figura 2: Estructuras de Laponita y Bentonita (Ochoa-Cornejo et al., 2014)

Las suspensiones de Laponita tienen, además, propiedades reológicas: para concentraciones de hasta aproximadamente el $3 \%$ en peso de agua, inicialmente se comportan como fluidos newtonianos. Sin embargo, después de 45 minutos, la solución de Laponita empieza a presentar un comportamiento de gel de tipo sólido (Bonn et al., 1999), capaz de resistir esfuerzos de corte, y con propiedades tixotrópicas (Willenbacher, 1996). Esta transición de solución a gel se refleja en el aumento del módulo de corte $G$ ' mostrado en la Figura 3 para una suspensión de Laponita al 3\%. El parámetro $G$ ' se obtiene de la medición de oscilaciones forzadas a pequeñas deformaciones, $y$ mide la componente elástica de la respuesta del material. Los datos con 4\% de Laponita en la Figura 3 muestran un aumento en $G^{\prime}$. Sin embargo, en muestras con $4 \%$ de Laponita, el comportamiento inicial de la suspensión es no newtoniano.

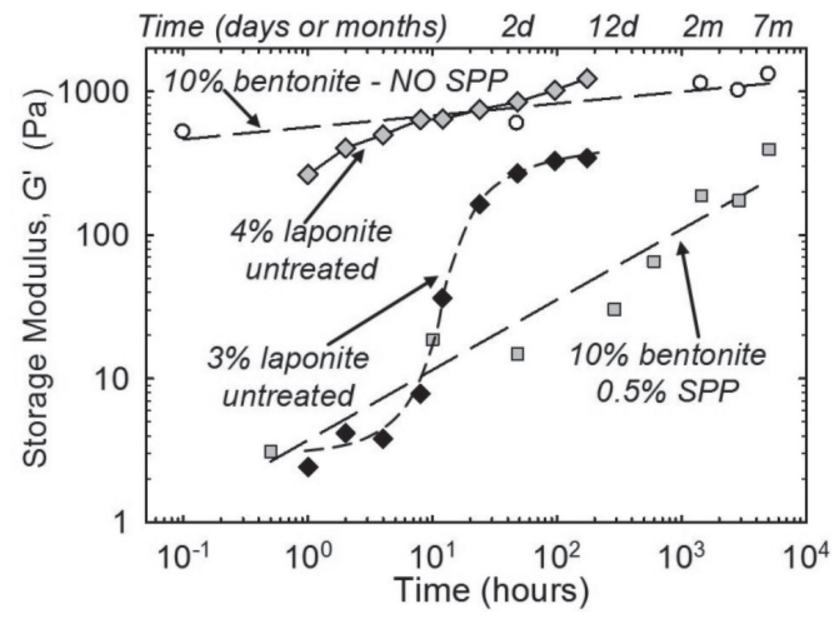

Figura 3: G' de Laponita y Bentonita (El Howayek, 2011)

La Figura 3 presenta datos de $G$ ' con el tiempo para dos suspensiones de Bentonita: una con $10 \%$ y otra con $10 \%$ de Bentonita con un $0.5 \%$ de pirofosfato de sodio SPP. El SPP retrasa la formación del gel de Bentonita, permitiendo el ingreso de la Bentonita en un medio poroso, como es descrito por El Mohtar et al. (2014). Notar que una concentración de $10 \%$ en peso de agua se traduce en aproximadamente $3 \%$ de Bentonita por peso seco de arena, cuando la suspensión se suministra en la matriz de arena. La Figura 3 también muestra que, después del tiempo de gelificación, las dos suspensiones de Laponita son reológicamente equivalentes (en términos de $G^{\prime}$ ) a la suspensión con $10 \%$ de Bentonita. Esto es significativo ya que demuestra que una suspensión con $10 \%$ de Bentonita dentro de los poros de una arena, tiene éxito en la prevención de la licuefacción (El Mohtar et al., 2013, 2014).

Este programa experimental con Laponita se centra en la resistencia cíclica de muestras de arena de Ottawa mezclados con Laponita, de manera que una suspensión con 3\% de Laponita quede dentro del espacio poroso de arena. Esta concentración corresponde a muestras de arena preparadas con $1 \%$ de Laponita con respecto al peso seco de la arena limpia.

\section{Preparación de las muestras y ensayos}

Las muestras se preparan en seco, mezclando arena limpia con $1 \%$ de polvo de Laponita (por peso seco de arena) en un cilindro de plástico equipado con un tubo de pluviación. 
Después, la mezcla se pluvia en el molde que se apoya en la base triaxial, controlando la altura de caída para lograr una densidad relativa en el intervalo de $15-25 \%$. Dos equipos triaxiales se utilizaron en esta investigación: el sistema CKC fabricado por Soil Testing Equipment, y el STX 050 fabricado por GCTS.

La densidad relativa de las muestras se calcula utilizando la relación de índice de vacíos intergranular, el cual considera el contenido fino como parte del espacio de vacío, y utiliza los valores de $e_{\max } \mathrm{y} e_{\min }$ de la arena limpia. Notar que el uso de un índice de vacíos constante permite comparaciones entre la arena limpia, y la misma arena en donde el espacio intersticial está siendo ocupado por partículas de Laponita. Tras la aplicación de una presión de cámara isotrópica inicial de 25 a $50 \mathrm{kPa}$, se pasa dióxido de carbono $\mathrm{CO}_{2}$ a través de la muestra, y luego agua deionizada y desaireada. A continuación se aplica una contrapresión entre 200 y $300 \mathrm{kPa}$ para asegurar la saturación, manteniendo constante la tensión efectiva. Para consolidar el suelo a $100 \mathrm{kPa}$, el valor mínimo aceptable del parámetro B es de 0.95 . Después de la consolidación, las muestras de arena limpias se someten inmediatamente al corte cíclico no drenado, mientras que las muestras de arena con Laponita se someten a un envejecimiento de 72 horas. Se debe tener en cuenta que 72 horas corresponde al tiempo cuando el módulo de corte de la suspensión con $3 \%$ de Laponita alcanza un valor relativamente constante, indicador que la gelificación de la suspensión de Laponita está completa (Figura 3). Además, a las 72 horas el módulo corte de la suspensión de Laponita es comparable a la de la suspensión con $10 \%$ de Bentonita, porcentaje que ha demostrado ser eficaz en el aumento de la resistencia de licuefacción de la arena (El Mohtar et al., 2013). El corte cíclico se realiza con una frecuencia de carga de $1 \mathrm{~Hz}$. Las razones de esfuerzo cíclico utilizadas oscilan entre $0.10 \mathrm{y}$ 0.14 para arena limpia, y entre 0.12 y 0.25 para arena con Laponita. Los ensayos se desarrollaron hasta alcanzar la pérdida completa de la presión de confinamiento efectiva en la muestra.

Los procedimientos descritos son similares a los seguidos en el trabajo de El Mohtar et al. (2014) para preparar muestras mezcladas en seco de Bentonita y arena Ottawa. Las diferencias entre los dos procedimientos se encuentran en la cantidad de material fino que se mezcla en peso seco con la arena (3\% de Bentonita, $1 \%$ Laponita) y la duración del envejecimiento antes del corte cíclico (72 horas para las muestras de arena con Laponita, y 24 ó 96 horas para muestras de arena con Bentonita). Otra diferencia está en la densidad relativa en los dos estudios: $15-25 \%$ para las muestras de arena con Laponita, y de 35 a $45 \%$ para las muestras de arena con Bentonita.

\section{Resultados del programa experimental}

La Figura 4 muestra los resultados de ensayos triaxiales cíclicos no drenados en arena limpia y de arena con $1 \%$ de Laponita, con densidad relativa en el intervalo de 15 $25 \%$. Se grafica el número de ciclos para la licuefacción versus la razón de corte cíclico aplicada. La flecha de la figura indica que la muestra no licúa en el número de ciclos indicado. Se observa la mejora alcanzada en la respuesta de arena limpia después de ser tratada con $1 \%$ de Laponita. Para similares niveles de CSR aplicado, el número de ciclos para llegar a la licuefacción aumenta en, al menos, dos órdenes de magnitud.

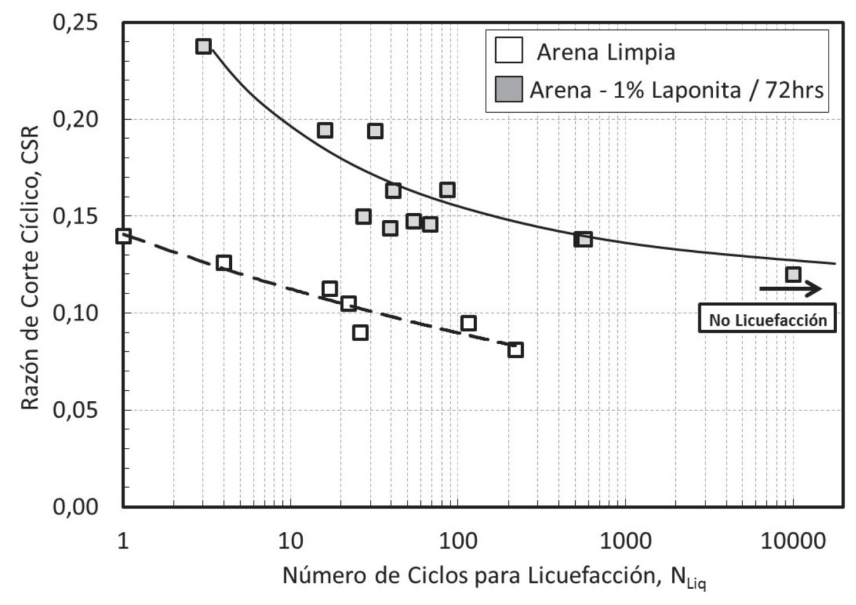

Figura 4: Resistencia cíclica de arena limpia, y arena con Laponita (1\%), densidad relativa $\operatorname{Dr}_{\text {sk }} \sim 15-25 \%$ (Ochoa-Cornejo et al., 2014, 2016; Ochoa-Cornejo, 2015)

La Figura 5 muestra resultados similares para muestras de arena limpia y muestras de arena con un 3\% de Bentonita. En este gráfico, las líneas presentadas son líneas de interpolación que se le aplicó a los datos experimentales. Las muestras tienen una densidad relativa en el rango de $30 \%-40 \%$. Se muestran dos grupos de datos para muestras de arena con Bentonita: uno con un período de envejecimiento (posterior a la consolidación) de 24 horas, y otro con un envejecimiento de 96 horas. Estos dos 


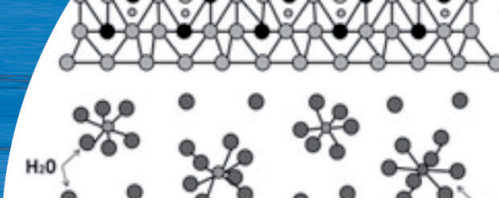

conjuntos de datos se muestran ya que incluyen el tiempo de envejecimiento de 72 horas utilizado para muestras de arena con Laponita. En la Figura 5, además, se observa que, tal como en el caso de Laponita, la adición de Bentonita aumenta la resistencia cíclica de la arena en un orden de magnitud con respecto a la arena limpia. También se aprecia que un aumento en el tiempo de envejecimiento induce un aumento adicional de la resistencia.

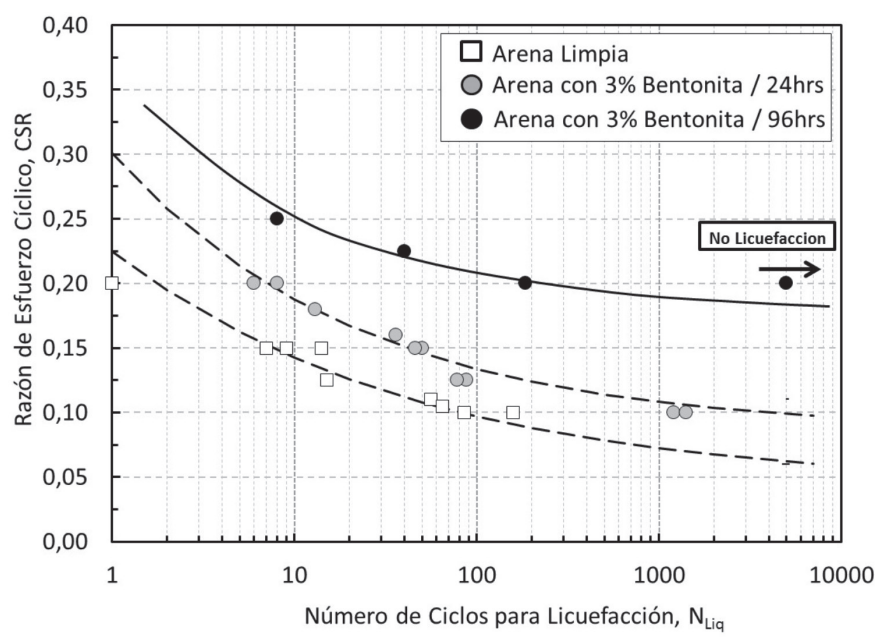

Figura 5: Resistencia cíclica de arena limpia, y de arena con Bentonita, densidad relativa $\operatorname{Dr}_{\mathrm{ck}} \sim 30-40 \%$ (El Mohtar et al., 2014; Ochoa-Cornejo et al., 2014, 2016; Ochoa-Cornejo, 2015)

Las Figuras 4 y 5 sugieren que la presencia de finos plásticos, en particular de alta plasticidad, dentro de una matriz de arena genera un efecto positivo sobre la resistencia a la licuefacción. También sugiere que la plasticidad de los finos juega un papel importante en la respuesta cíclica del suelo, ya que la adición de 1\% de Laponita logra una mejora similar a la conseguida con $3 \%$ de Bentonita. La mejora en la resistencia a la licuefacción observada con la adición de una nano-arcilla altamente plástica es causada por un retraso del exceso de presiones de poros durante la carga cíclica. Los resultados y observaciones indican que este retraso es el resultado de la interacción entre los granos de arena y el fluido intersticial de tipo sólido formado por los finos plásticos y el agua en el espacio de los poros, lo que reduce la movilidad de los granos de arena, retrasando la generación del exceso de presiones de poros, por ende, aumentando la resistencia cíclica del suelo.

Esta hipótesis está apoyada por la observación directa de la microestructura de las muestras de arena con Bentonita, y de arena con Laponita, utilizando microscopía electrónica
cryo-SEM. Esta técnica implica la sublimación de las muestras a temperaturas muy bajas $\left(-85^{\circ} \mathrm{C}\right)$, y la formación de imágenes en condiciones criogénicas $\left(-130^{\circ} \mathrm{C}\right)$, de modo que la muestra permanece cerca de su estado natural, evitando deshidratación. La Figura 6(a) y 6(b) presentan imágenes de la microestructura de una muestra de arena con Bentonita, y una muestra de arena con Laponita, respectivamente. Ambas imágenes muestran que los granos de arena están rodeados por una densa matriz celular que corresponde al gel formado en el espacio intersticial, por la hidratación de la Bentonita o la Laponita. Este fluido en los poros se caracteriza por un comportamiento de tipo sólido. Sus propiedades (por ejemplo, $G$ ' en la Figura 2) aumentan la respuesta elástica del sistema, reduciendo las deformaciones plásticas, reduciendo el exceso de presiones de poros.

a)

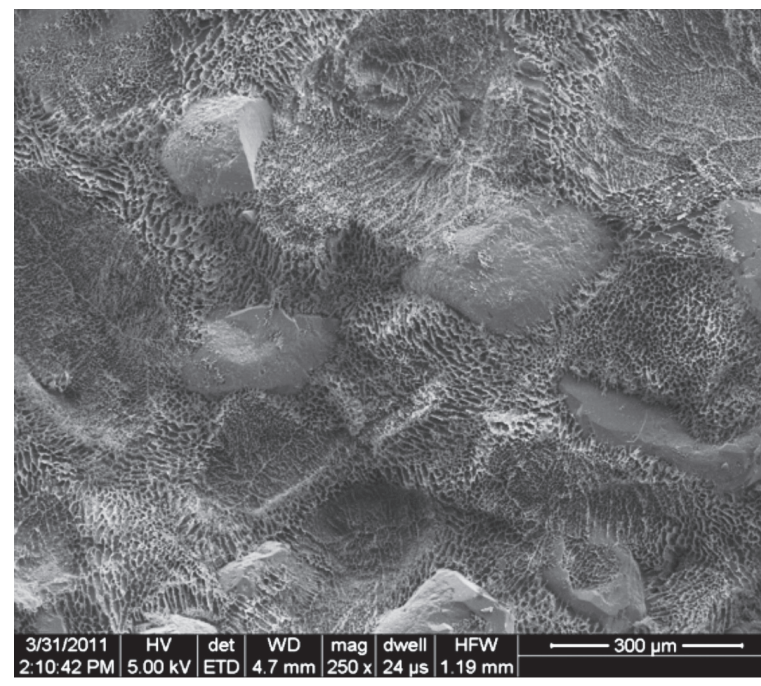

b)

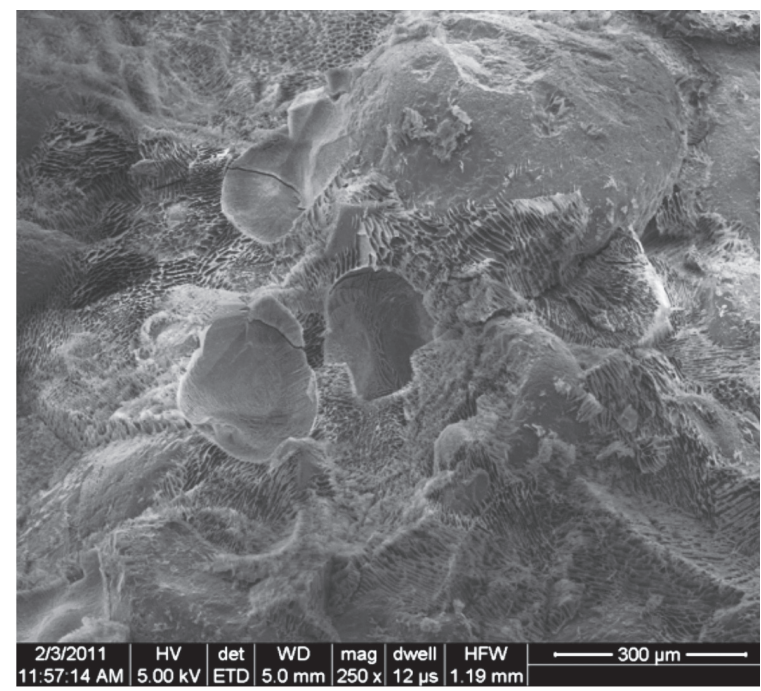

Figura 6: Fotografías SEM de arenas con: a) 3\% de Bentonita y b) 1\% de Laponita (Ochoa-Cornejo et al., 2014, 2016) 


\section{Conclusiones}

Este trabajo presenta los resultados experimentales de triaxiales cíclicos ejecutados en arenas con finos de alta plasticidad, y evalúa la mejora en la resistencia a la licuefacción, fenómeno de gran relevancia para la ingeniería chilena. En particular, el objetivo del trabajo fue evaluar el impacto de la adición de 1\% de Laponita en la respuesta cíclica no drenada de la arena, y comparar estos resultados con los de arena limpia, así como los de arena tratada con Bentonita. Este trabajo experimental se realizó en muestras de arena con 1\% de Laponita, mezcladas en seco. Los resultados muestran que la presencia de $1 \%$ Laponita aumenta significativamente el número de ciclos necesarios para llegar a la licuefacción, en comparación con la arena limpia en las mismas condiciones de densidad y corte solicitante. La mejora es comparable a la obtenida utilizando Bentonita. Sin embargo, consistente con la mayor plasticidad de la Laponita, en relación con Bentonita, se necesita una menor cantidad de Laponita para lograr un grado de mejora equivalente. El aumento de la resistencia cíclica se atribuye al gel formado en el interior del espacio intersticial, como resultado de la hidratación de la Laponita. El fluido tixotrópico formado tiene un comportamiento de tipo sólido, reduciendo la movilidad de las partículas de arena, retardando la generación del exceso de presiones de poros y por ello aumentando la resistencia a la licuefacción. El trabajo proporciona evidencia de que la Laponita tiene gran potencial para mitigar la licuefacción de arenas.

\section{Agradecimientos}

Se agradece el apoyo de NSF Foundation, Purdue University, y Universidad de Chile.

\section{Referencias}

Bonn, D., Kellay, H., Tanaka, H., Wegdam, G. and Meunier, J. (1999). Laponite: What is the difference between a gel and a glass?. Langmuir 15(22), 7534-7536

Clarke, J.P. (2008). Investigation of time-dependent rheological behavior of Sodium Pyrophosphate-Bentonite suspensions. MSc thesis, Purdue University, West Lafayette, Indiana

El Howayek, A. (2011). Characterization, rheology and microstructure of laponite suspensions. MSc thesis, Purdue University, West Lafayette, Indiana
El Mohtar, C.S., Bobet, A., Drnevich, V.P., Johnston, C.T. and Santagata, M.C. (2014). Pore pressure generation in sand with bentonite: from small strains to liquefaction. Géotechnique 64(2), 108-117

El Mohtar, C.S., Bobet, A., Santagata, M.C., Drnevich, V.P. and Johnston, C.T. (2013). Liquefaction mitigation using bentonite suspensions. Journal of Geotechnical and Geoenvironmental Engineering 139(8), 1369-1380

Gallagher, P.M. and Mitchell, J.K. (2002). Influence of colloidal silica grout on liquefaction potential and cyclic undrained behavior of loose sand. Soil Dynamics and Earthquake Engineering 22(9), 1017-1026

Ishihara, K. (1993). Liquefaction and flow failure during earthquakes. Géotechnique 43(3), 351-415

Ishihara, K. and Koseki, J. (1989). Discussion On the cyclic shear strength of fines containing sands. Proceedings of the Eleventh International Conference on Soil Mechanics and Foundation Engineering, Rio de Janiero, Brazil, 101-106

Koester, J.P. (1994). The influence of fines type and content on cyclic strength. Proceedings of the ASCE National Convention on Ground Failures under Seismic Conditions, Prakash and Dakoulas eds., 17-33

Mongondry, P., Nicolai, T. and Tassin, J.F. (2004). Influence of pyrophosphate or polyethylene oxide on the aggregation and gelation of aqueous laponite dispersions. Journal of Colloid and Interface Science 275(1), 191-196

Mooney, R.W., Keenan, A.G. and Wood, L.A. (1952). Adsorption of water vapor by Montmorillonite. I. Heat of desorption and application of BET theory1. Journal of the American Chemical Society 74(6), 1367-1371

Ochoa-Cornejo, F.A. (2015). Cyclic behavior of sands with superplastic fines. $\mathrm{PhD}$ thesis, Purdue University, West Lafayette, Indiana

Ochoa-Cornejo, F., Bobet, A., Johnston, C.T., Santagata, M. and Sinfield, J.V.(2016). Cyclic behavior and pore pressure generation in sands with laponite, a super-plastic nanoparticle. Soil Dynamics and Earthquake Engineering 88, 265-279

Ochoa-Cornejo, F., Bobet, A., Johnston, C., Santagata, M. and Sinfield, J. (2014). Liquefaction 50 years after Anchorage 1964; how nanoparticles could prevent it. Proceedings of the $10^{\text {th }}$ National Conference in Earthquake Engineering, Earthquake Engineering Research Institute, Anchorage, USA 
Polito, C.P. and Martin, J.R. (2001). Effects of nonplastic fines on the liquefaction resistance of sands. Journal of Geotechnical and Geoenvironmental Engineering 127(5), 408-415

Ruzicka, B. and Zaccarelli, E. (2011). A fresh look at the Laponite phase diagram. Soft Matter 7(4), 1268-1286

Troncoso, J. and Verdugo, R. (1985). Silt content and dynamic behavior of tailings sands. XI International Conference on Soil Mechanics and Foundation Engineering, San Francisco, 13111314

Willenbacher, N. (1996). Unusual thixotropic properties of aqueous dispersion of Laponite RD. Journal of Colloid and Interface Science 182(2), 501-510
Youd, T.L., Idriss, I.M., Andrus, R.D., Arango, I., Castro, G., Christian, J.T., Dobry, R., Liam Finn, W.D., Harder, L.F., Hynes, M.E., Ishihara, K., Koester, J.P., Liao, S.S.C., Marcuson, W.F., Martin, G.R., Mitchell, J.K., Moriwaki, Y., Power, M.S., Robertson, P.K., Seed, R.B. and Stokoe, K.H. (2001). Liquefaction resistance of soils: summary report from the 1996 NCEER and 1998 NCEER/NSF workshops on evaluation of liquefaction resistance of soils. Journal of Geotechnical and Geoenvironmental Engineering 127(10), 297-313 